\title{
Sustainable Energy Harvesting System Utilizing Fluid- elastic Vibration of a Tube Array due to Ocean Flow
}

\author{
Tomohiro Ito ${ }^{1}$, Atsuhiko Shintani ${ }^{1}$, Chihiro Nakagawa ${ }^{1}$
}

\begin{abstract}
Various types of sustainable energy harvesting systems have been developed and already in commercial use. After the accident in Fukushima nuclear power plant, these sustainable energy systems are becoming more important. But some of them such as wind turbine system deeply depend on the climate, and some types that utilize a turbine operating in the sea water have problems of corrosion or maintenance.

Thus, we developed a new energy harvesting system that utilizes "Fluid-elastic Vibration of a Tube Array" due to ocean flow. This system does not depend on the climate change and the generator is placed above sea surface.

In this paper, first, a theoretical study on the energy harvesting due to fluid-elastic vibration of a tube array was conducted in order to confirm the feasibility on the energy harvesting due to the proposed system. And secondly, a small scale test model was fabricated that utilized air flow instead of water flow, for convenience. Tests were conducted in the laboratory. The relationship between the flow rate and generated electric power were obtained for various tube array patterns.

From both the theoretical and experimental studies, we confirmed that the proposed system could be one of the sustainable energy harvesting systems. In addition, the most efficient tube array as for energy harvesting was discussed.
\end{abstract}

Key Words: Sustainability, Renewable energy, Energy harvesting system, Fluid-elastic vibration, Tube array, Ocean flow

\section{Introduction}

Up to now, a lot of types of renewable energy harvesting systems, such as wind power, solar power, geothermal power, or ocean power, have been developed and are already in commercial use. On the other hand, currently, huge floods and hurricanes occurred all over the world, which are believed to be caused by the global warming brought by the increase in carbon dioxide generated by the combustion of fossil fuel. In addition, the accident in the Fukushima Daiichi nuclear power plant that occurred in 2011 in Japan reduces the dependence on the nuclear power. Thus, the above mentioned renewable energy systems are becoming more important in order to maintain the global safety. However, the capacities of the above mentioned systems are rather smaller than the conventional power plants such as thermal power or nuclear power systems. In addition, some of them depend on the climate and some are immersed in the sea water and require special attention in maintenance. Thus, it is meaningful to develop a new renewable energy harvesting system to expand a variety.

Among the above-mentioned natural energy resources, we focused on the tidal power. Up to now, various types of energy harvesting systems utilizing ocean power have been 
developed [1]. Some utilize the difference in the sea surface level due to tide. Some utilizes the sea surface fluctuation, e.g., Kanki [2] developed a system that utilizes a gyro and the ship rotation due to sea surface fluctuation. And Kyozuka [3] utilizes a water turbine operated by ocean flow. Here, the authors developed a new energy harvesting system driven by the ocean current that does not depend on the climate and has huge power. In this system, fluid-elastic vibration of a tube array due to ocean current is utilized. As well known, when the tube array is subjected to fluid cross flow and the flow velocity becomes enough large, the tube array reveals fluid-elastic vibration in which the tube array vibrates with very large amplitude leading to the failure of the tubes at worst. Therefore, up to now, many researchers have ever focused on the mitigation of fluidelastic vibration [4]. One of the authors [5] also has ever conducted researches on the mitigation of fluid-elastic tube vibration due to cross flow jetted from narrow slot. However, from the other viewpoint, the tubes revealing fluid-elastic vibration are believed to have very large kinetic energy. Thus, the authors developed a new energy harvesting system that utilizes fluid-elastic vibration of a tube array due to fluid flow, and confirmed the feasibility of the proposed system experimentally [6].

In this study, the possibility of energy harvesting due to the proposed system was studied theoretically, and in addition, the effects of the tube array patterns on the generated energy were investigated by using a fundamental test apparatus that simulates fluid-elastic vibration of a tube array, in which air flow was used instead of water flow for convenience. The relationship between the flow velocity and generated electric power was investigated for various tube array patterns. As a result, it was found that the proposed energy harvesting system was feasible and could generate electric power. In addition, the most suitable tube array pattern and the future tasks that are required for the development of the actual systems were clarified.

\section{Energy harvesting system by fluid-elastic vibration of a tube array}

The authors developed an energy harvesting system utilizing fluid-elastic vibration of a tube array due to fluid cross flow. Tubes will vibrate with very large amplitudes when fluid-elastic vibration occurs.

\subsection{Fluid-elastic vibration of a tube array without energy harvesting system}

Fig. 1 shows the outline of fluid-elastic vibration of a tube array subjected to cross flow. As shown in this figure, when a tube array is subjected to fluid cross flow with increasing flow velocity, vibration amplitude of the tube array becomes large according to the increase in flow velocity. In relatively small flow velocity region, the vibration amplitude shows a small peak that is thought to be caused by vorticity-induced vibration. And for the flow velocity region larger than the critical flow velocity $U_{\text {cr }}$, fluidelastic vibration occurs suddenly accompanied with very large amplitude which can become infinity theoretically. Connors [7] derived the equation that gives the critical flow velocity $U_{\text {cr }}$ for the tube without energy harvesting system by considering the input energy $W$ due to fluid flow and the dissipated energy $E$ due to damping force. It is assumed that when $W$ becomes larger than $E$, fluid-elastic vibration occurs. Thus, $W=E$ gives the critical flow velocity $U_{\text {cr }}$. 
Figure 2 shows an analytical model of a tube array vibrating in the fluid flow. Fig. 2

(a) illustrates the outline of a tube array subjected to fluid cross flow and Fig. 2 (b) does an analytical model of tube vibration. A single tube vibration due to fluid excitation force is depicted here. This tube is assumed to vibrate both in the drag direction (=x direction) and the lift direction ( $=y$ direction) due to fluid forces both in the drag and lift directions. Fluid force is assumed to be in the sinusoidal form, and so is the tube vibration. And both of them are assumed to be in resonance condition, thus are expressed as follows:

$$
\begin{aligned}
F_{D} & =F_{D 0} \cos \omega t \\
F_{L} & =F_{L 0} \cos \omega t \\
x & =x_{0} \sin \omega t \\
y & =y_{0} \sin \omega t
\end{aligned}
$$

Here, $x_{0}, y_{0}$ are displacement amplitudes, and $F_{D 0}, F_{L 0}$ are fluid force amplitudes expressed by the following equations.

$$
\begin{aligned}
& F_{D 0}=\frac{1}{2} C_{D} \rho U^{2} D l \\
& F_{L 0}=\frac{1}{2} C_{L} \rho U^{2} D l
\end{aligned}
$$
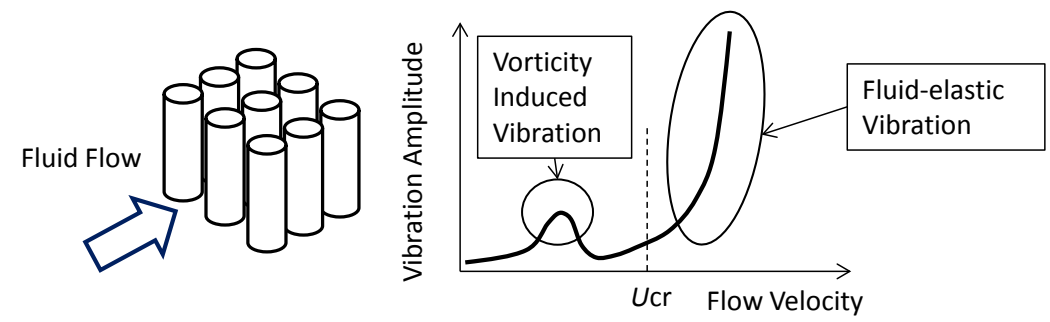

Fig. 1 Outline of fluid-elastic vibration of a tube array subjected to cross flow

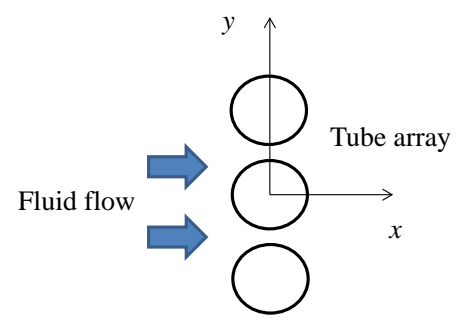

(a) Tube array subjected to fluid cross flow

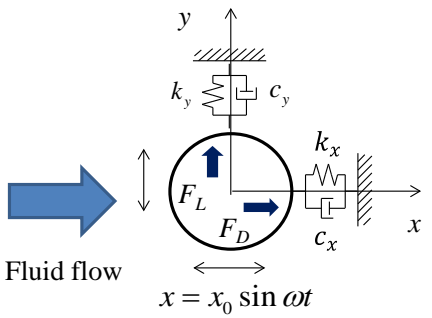

(b) Analytical model of tube vibration

Fig. 2 Analytical model of a tube array vibrating in the fluid flow

Where, $C_{D}, C_{L}, \rho, U, l$ are the drag and lift coefficients, fluid density, flow velocity and 
the tube length, respectively. Using these definitions, the input energy into the tube from fluid flow can be obtained by the following time integration during a period of $T$.

$$
\begin{aligned}
& W_{D}=\int_{0}^{T} F_{D} d x \\
& W_{L}=\int_{0}^{T} F_{L} d y
\end{aligned}
$$

Substituting Eqs. (1) (3) into Eq. (4), $W_{D}, W_{L}$ are obtained as,

$$
\begin{aligned}
& W_{D}=\frac{\pi}{2} C_{D} \rho U^{2} D l x_{0} \\
& W_{L}=\frac{\pi}{2} C_{L} \rho U^{2} \text { Dly }_{0}
\end{aligned}
$$

While, the dissipated energy by the damper will be given as follows:

$$
\begin{aligned}
& E_{D}=\int_{0}^{T} c_{x} \dot{x} d x=\pi c_{x} x_{0}{ }^{2} \omega_{n}=\delta m x_{0}{ }^{2} \omega_{n}{ }^{2} \\
& E_{L}=\int_{0}^{T} c_{y} \dot{y} d y=\pi c_{y} y_{0}{ }^{2} \omega_{n}=\delta m y_{0}{ }^{2} \omega_{n}{ }^{2}
\end{aligned}
$$

Here, $c_{x}, c_{y}, \omega_{n}, \delta$ are damping coefficients in $\mathrm{x}$ and $\mathrm{y}$ directions, natural circular frequency and logarithmic decrement of the tube, respectively. When the input energy exceeds the dissipated energy, fluid-elastic vibration occurs. Then, the following equation gives the onset condition of fluid-elastic vibration.

$$
W_{D}+W_{L}=E_{D}+E_{L}
$$

Substituting Eqs. (5) and (6) into Eq. (7), and introducing the mass per unit length $M$ and the natural frequency $f_{\mathrm{n}}$ of the tube, the critical flow velocity is derived as follows.

$$
\frac{U}{f_{n} D}=K_{c r} \sqrt{\frac{M \delta}{\rho D^{2}}}
$$

In deriving Eq. (8), the drag and lift coefficients are assumed to be proportional to the displacement amplitude of the tube, and the displacement in the lift direction is assumed to be proportional to that in the drag direction. Here, $K_{c \mathrm{r}}$ is a constant value which is decided based on a lot of experimental data. Connors [7] gives the lowest value of 2.9.

\subsection{Proposed energy harvesting system utilizing fluid-elastic vibration}

The authors developed an energy harvesting system that utilizes fluid-elastic vibration of a tube array. Fig. 3 shows the outline of the tube arrangement and energy harvesting system. As shown in this figure, each tube is supported by a rigid beam with a pin joint at its top end. Here, the tube motion in the drag direction is constrained, because it is known that the motion in the drag direction is smaller than that in the lift direction. At this pin joint, a leaf spring is attached to provide the restoring moment to the rigid beam. In this sense, the tube reveals pendulum type motion. In order to convert the pendulum type motion to the rotation of a rod, a rack and pinion system is introduced. The rod rotation is changed into electric power by using electro-magnetic induction principle.

\subsection{Theory on fluid-elastic vibration for pendulum type tube motion}

In deriving Eq. (8), the tube is assumed to vibrate translationally. But, in the present system, the tubes reveal pendulum type motions as stated before. Thus, the 
horizontal displacement of the tube in the $y$ direction changes according to the location $z$ in the longitudinal axis as illustrated in Fig. 4. When the tube rotation angle is expressed as $\theta=\theta_{0} \sin \omega t$, the horizontal displacement $y$ can be expressed as;

$$
y=(l-z) \sin \theta=(l-z) \sin \left(\theta_{0} \sin \omega t\right)
$$

Considering the rotational motion of the tube, similar manipulations as shown in section 2.1 gives a formulation of the critical flow velocity for the pendulum type tube motion as;

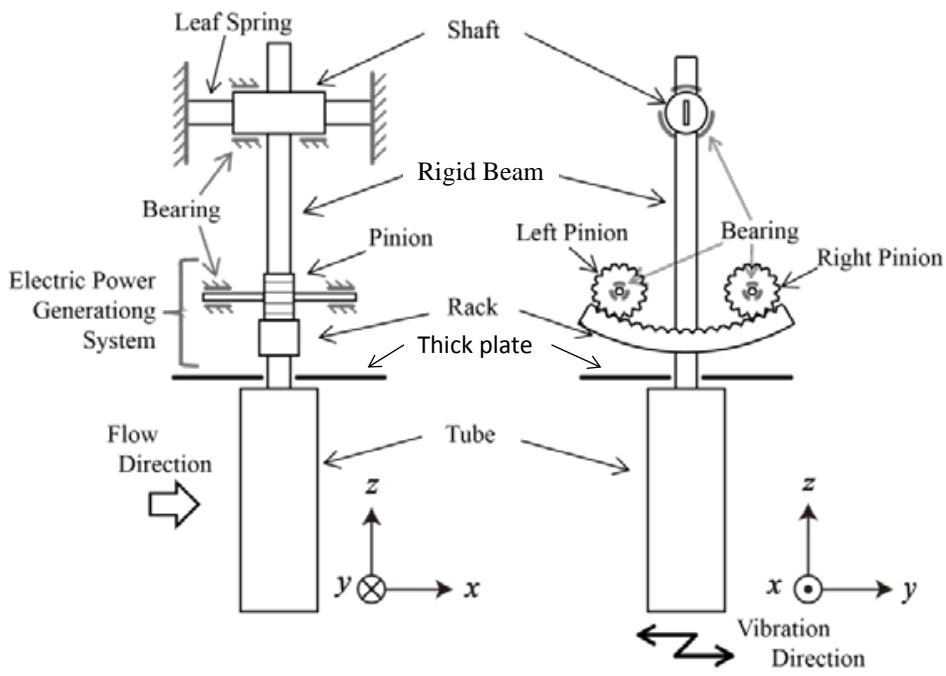

Fig. 3 Proposed energy harvesting system and the outline of a tube arrangement

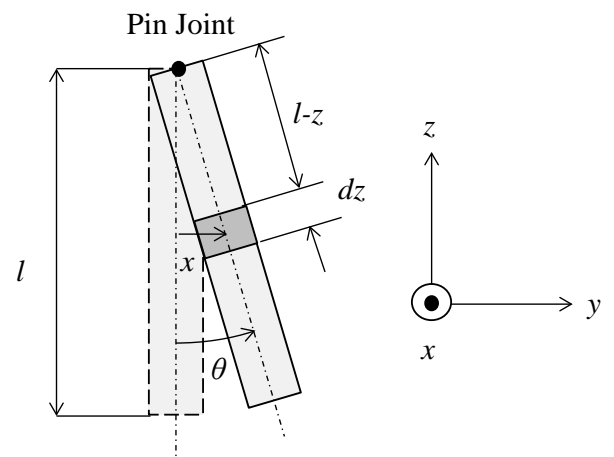

Fig. 4 Outline of pendulum type motion of proposed tube arrangement

$$
\frac{U}{f_{\theta} D}=K_{c r} \sqrt{\frac{M \delta}{\rho D^{2}}}
$$


Here, $f_{\theta}$ is the natural frequency of pendulum motion. The details of the manipulation are slightly complicated but not shown here due to the limitation of paper pages. Eq. (10) is very similar as Eq. (8), except for that $K_{\text {cr }}$ here includes the Vessel function of the first order, in spite that $K_{\mathrm{cr}}$ will be decided experimentally in the actual plants.

\subsection{Theory on fluid-elastic vibration of a tube array with energy harvesting system}

In deriving Eq. (10), the tube array is assumed not to have an energy harvesting system. However, when the tube array has an energy harvesting system, energy balance will change. A part of the input energy will be consumed as the generated electric energy $E_{\mathrm{G}}$. Thus, the energy balance will become as follows:

$$
W_{D}+W_{L}=E_{D}+E_{L}+E_{G}
$$

Assuming an electric power generation efficiency $\eta_{E}, E_{\mathrm{G}}$ will be given as,

$$
E_{G}=\eta_{E} \frac{1}{2} I \omega_{\theta}^{2}
$$

Where, I and $\omega_{\theta}$ are rotational inertia moment and natural circular frequency of the tube, respectively. Here, we introduce a "coefficient of electric power generation" $\gamma_{G}$ defined by the following equation.

$$
\gamma_{G}=\frac{E_{G}}{I \omega_{\theta}^{2}\left(1+\alpha^{2}\right) \theta_{0}^{2}}
$$

Where, $\alpha$ denotes the ratio between the amplitudes in the drag and lift directions. From Eq. (13), $E_{\mathrm{G}}$ will be shown as,

$$
E_{G}=\gamma_{G} I \omega_{\theta}^{2}\left(1+\alpha^{2}\right) \theta_{0}^{2}
$$

Substituting Eq. (14) into Eq. (11) and conducting similar manipulations as in the section 2.3 , the formulation that expresses the critical flow velocity for the tube array equipped with an energy harvesting system will be obtained as follows.

$$
\frac{U}{f_{\theta} D}=K_{c r} \sqrt{\frac{M\left(\delta+\gamma_{G}\right)}{\rho D^{2}}}
$$

This equation is called as "Equation of fluid-elastic vibration with energy harvesting". It is noted that the energy harvesting by the proposed energy harvesting system corresponds to the increase in the logarithmic decrement, i.e., the increase in the damping ratio of the tune array.

\section{Experimental methods for electric power generation test}

\subsection{Test apparatus}

A fundamental test apparatus that simulates the tube array subjected to fluid flow is fabricated. Figs. 5 and 6 illustrate the outline and the photo of the flow channel, respectively. This flow channel was also used in the previous study [6] in order to investigate the feasibility of the proposed electric power generation system. The flow channel made by transparent vinyl chloride has a dimension of $144[\mathrm{~mm}]$ in width, 110 $[\mathrm{mm}]$ in height and $1[\mathrm{~m}]$ in length. Air is used as fluid for convenience. An air blower is settled at the exit of the flow channel for sucking air. The air flow enters into the flow 
channel from the left end of the channel. This manner is often used in order to reduce turbulence in the air flow. The dimensions of the flow channel is decided by considering the air blower specifications; maximum flow rate of $19.6\left[\mathrm{~m}^{3} / \mathrm{min}\right]$ and maximum head of $13.8[\mathrm{kPa}]$. The tube array is also made by vinyl chloride, and installed into this flow channel. In the present study, the maximum tube number is settled to 6 while 9 in the previous study [6]. The purposes of this study are (1) to evaluate the minimum tube number which is required for fluid-elastic vibration with energy harvesting system and (2) to evaluate the effects of the surrounding tube vibration condition. Thus, the tube number is reduced to 6. Figs. 7 and 8 illustrate the photo of $3 \times 3$ tube array and the top view of the $2 \times 3$

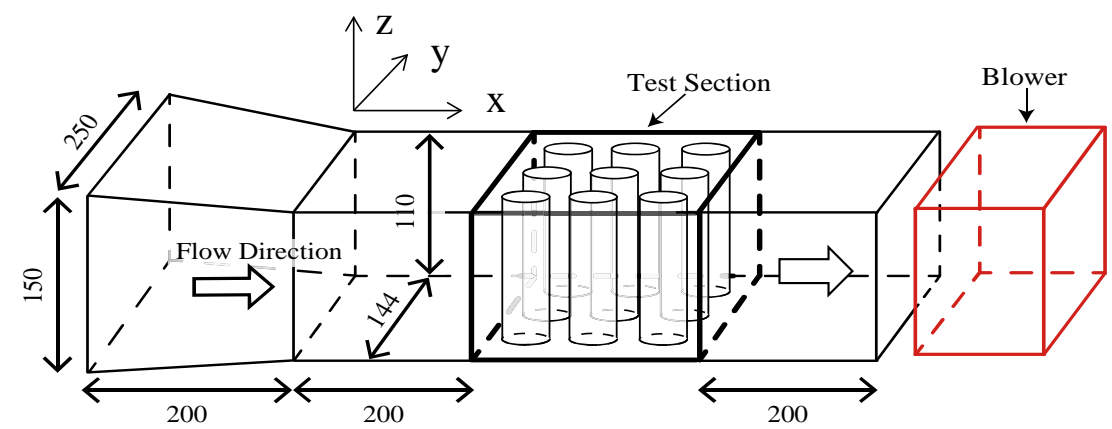

Fig. 5 Outline of flow channel

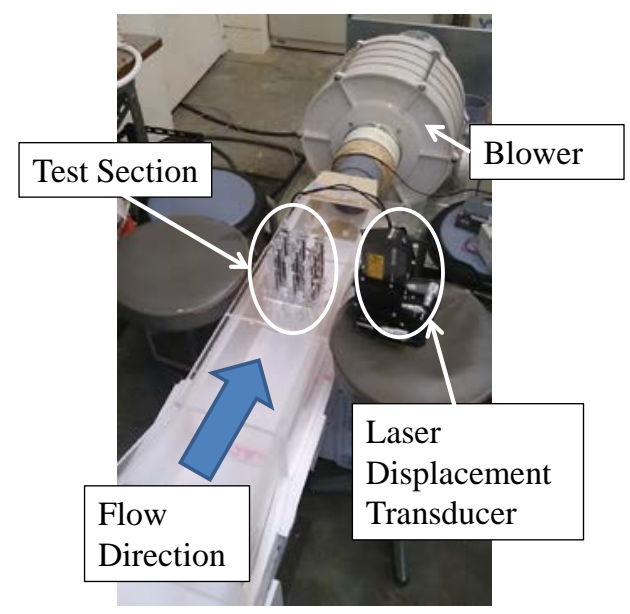

Fig. 6 Photos of a flow channel

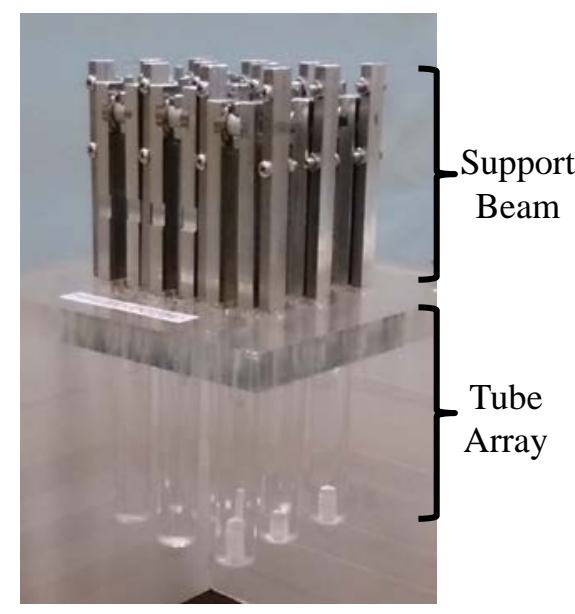

Fig. 7 Photo of tube array

and $1 \times 3$ tube array, respectively. As shown in Fig. 8 , in order to simulate the wall conditions, half cylindrical tubes are attached to both side walls of the channel. The tube dimensions are $20[\mathrm{~mm}]$ in diameter, $90[\mathrm{~mm}]$ in length and the mass per unit length is $0.377[\mathrm{~kg} / \mathrm{m}]$. As stated in the previous chapter, this tube is fixed to the bottom end of a light rigid beam made by aluminum and this rigid beam is supported at the top end using tiny bearings. A leaf spring is used to apply rotational moment to the rigid beam. Thus, 
the tubes fabricated here will reveal rotational motion. The shear modulus of the leaf spring is $27[\mathrm{GPa}]$. In order to convert this rotational motion into the rotation of the shaft and to harvest electricity, a rack and pinion system is introduced as shown in Fig. 3. From the shaft rotation, electric power is harvested utilizing electromagnetic induction principle. The displacement of the tube is measured by using a laser displacement transducer. The displacement of the top end of the rigid beam is measured from which the tube displacement is calculated. Electric power is calculated from the generated voltage and resistance. Flow velocity through the tube gap is obtained by dividing the flow rate with the cross sectional area.

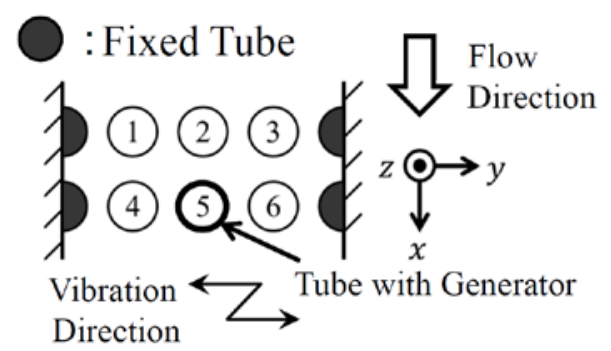

(a) $2 \times 3$ tube array

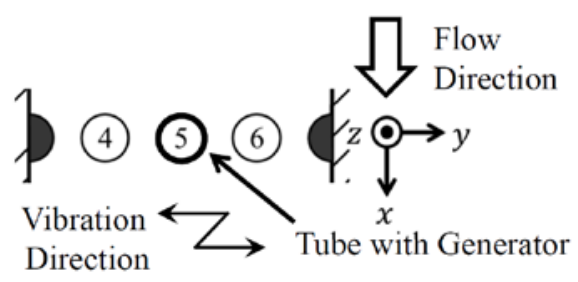

(b) $1 x 3$ tube array

Fig. 8 Tube arrays used in the present tests

\subsection{Test condition}

In this study, the flow velocity is increased step by step, and the tube vibration amplitude and the generated electric power are measured. Two kinds of tube array, $2 \times 3$ and $1 \times 3$, are employed as illustrated in Fig. 8. The electric power generator system (hereafter, called a generator) is attached to the No. 5 tube. In order to evaluate the effects of the surrounding tube vibration, the surrounding tubes are clamped in several patterns, as shown in Table 1. In the case 1, all of the surrounding tubes are free to vibrate. In the case 2, No. 1, 2 and 3 are clamped, and No. 1 and 3 in the case 3, No. 1 and 4 in the case 4 , No. 1, 3, 4 and 6 in the case 5 . In the case $6,1 \times 3$ tube array is tested in order to compare the results with that of the case 2.

Table 1 Tube array patterns

\begin{tabular}{lll}
\hline Case No. & Tube array & Clamped tubes \\
\hline 1 & $2 \times 3$ & - \\
\hline 2 & $2 \times 3$ & No. $1,2,3$ \\
\hline 3 & $2 \times 3$ & No. 1,3 \\
\hline 4 & $2 \times 3$ & No. 1,4 \\
\hline 5 & $2 \times 3$ & No. $1,3,4,6$ \\
\hline 6 & $1 \times 3$ & - \\
\hline
\end{tabular}




\section{Test results on generated electric power}

\subsection{In the case of $2 \times 3$ tube array without clamped tubes (Case 1)}

Before conducting the flow-induced vibration tests, the vibration characteristics, such as natural frequency and damping ratio are measured. Natural frequencies are ranging from 2.3 to $2.9 \mathrm{~Hz}$, while the damping ratios are from 3.8 to $4.9 \%$. Damping ratio of No. 5 tube to which a generator is attached is around $15 \%$, larger than those of the other tubes as anticipated.

Displacement amplitude for each tube is measured, and generated electric power is obtained from the measured voltage. Fig. 9 illustrates the typical time histories of displacement and generated voltage of No. 5 tube in the case 1 for flow velocities of $\mathrm{U}=10.9[\mathrm{~m} / \mathrm{s}]$ and $\mathrm{U}=14.2[\mathrm{~m} / \mathrm{s}]$. Fig. 10 shows the relationship between the displacement amplitude and the flow velocity. In Fig. 10, vertical bars stand for the range of displacement. It is known that both the tube displacement and generated voltage increase suddenly when the flow velocity becomes large, although generated voltage are very small and fluctuating. In addition, downstream tubes tend to show larger displacement amplitude. It is thought that the upstream tubes tend to suppress flow turbulence and enhance vibration of downstream tubes.
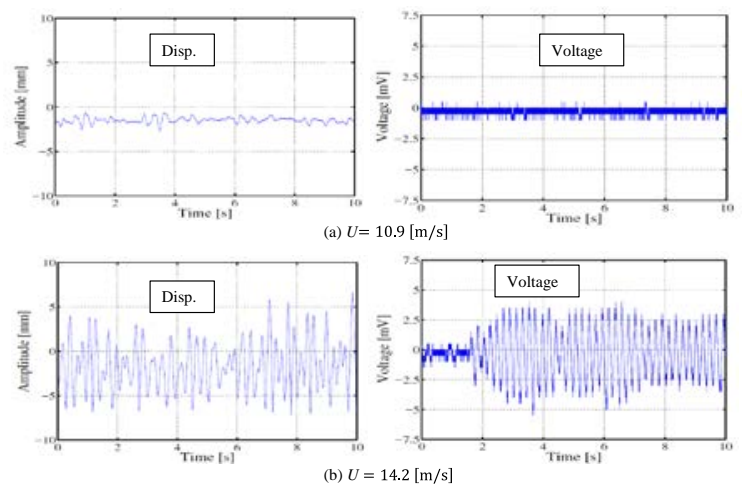

Fig. 9 Typical time histories of displacement and voltage of No. 5 tube in the case 1 (2x3 array) (a) $U=10.9$ $[\mathrm{m} / \mathrm{s}]$, (b) $U=14.2[\mathrm{~m} / \mathrm{s}]$
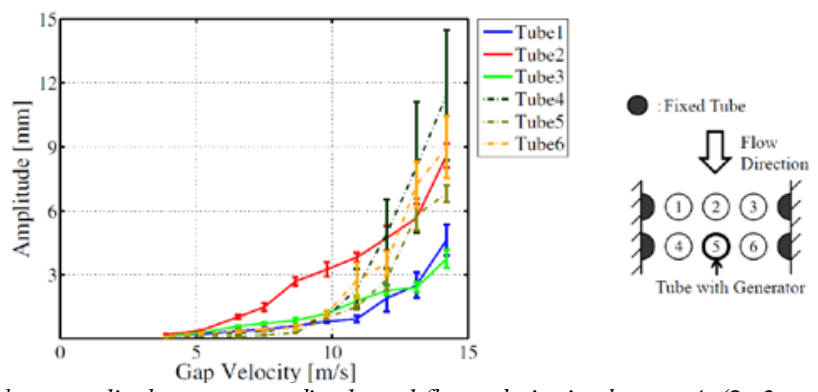

Fig. 10 Relationship between displacement amplitude and flow velocity in the case 1 (2x3 array) 


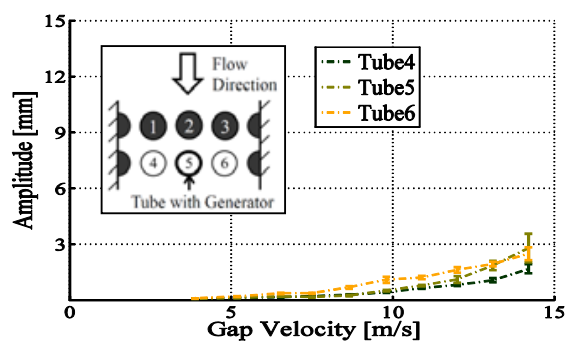

(a) Case 2

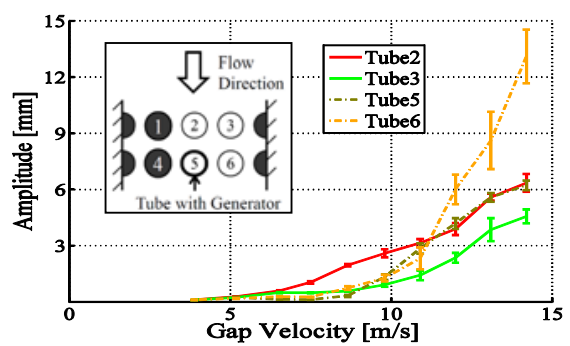

(c) Case 4

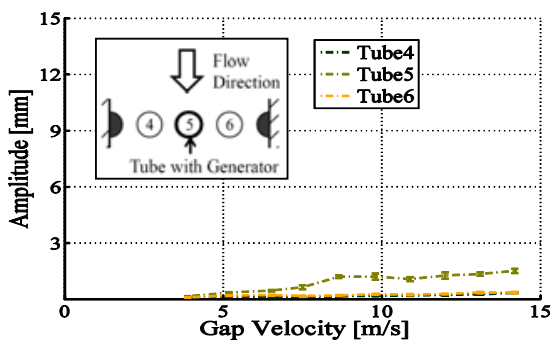

(e) Case 6

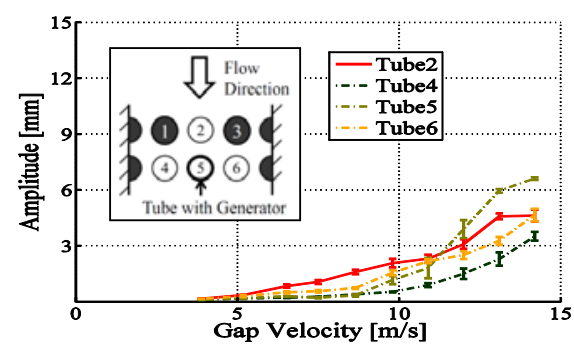

(b) Case 3

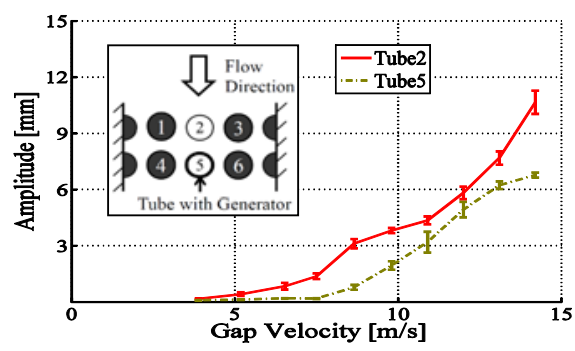

(d) Case 5

: Clamped Tube

Fig. 11 Relationship between displacement amplitude and flow velocity (Case 2 to Case 6)

\subsection{In the case of $2 \times 3$ tube array with clamped tubes (Case 2 to Case 6)}

Similar results with the case 1 are obtained for the cases 2 to 6 . Only the relationship between the displacement and flow velocity is shown here as depicted in Fig. 11. It is known that the cases 4 and 5 show larger amplitude than the other cases, while the cases 2, 3 and 6 show rather small amplitude. Thus, it can be said that the upstream tubes have large influences to the vibration amplitude of the downstream tubes. Clamping all of the upstream tubes or removing them will reduce vibration of the tube array. Fluid-elastic vibration is caused by the fluid-structure interaction of the tube array in nature, thus some amount of tube number seems to be needed for the occurrence of fluid-elastic vibration.

\section{Discussion on the generated electric power}

The relationship between the generated electric power by No. 5 tube and flow 
velocity is summarized in Figs. 12 and 13. Fig. 12 discusses the effect of the upstream tubes, and Fig. 13 does the effect of the tubes in the lift direction. It is obvious, from Fig. 12, that the case 1 gives the largest generated electric power than those in the cases 2 and 6 . Thus, it can be said that the upstream tubes are better to be installed and not to be clamped. From Fig. 13, it is noted that the case 5 gives largest generated electric power, although the differences between three cases are not so large. It is thought that the symmetric tube array patterns had better to be employed in order to obtain larger vibration amplitude, which subsequently leads to larger generated electric power. The previous test results by the authors [6] indicates that 3x3 tube array gives 1.5 times larger generated electric power than that by $2 \times 3$ tube array. Therefore, results of the case 5 are very convenient for the actual system in the view point of construction cost reduction. Only two vibrational tubes are required and the surrounding tubes are simply to be clamped.

From these results, it is found that the proposed energy harvesting system could generate electric power, although the power is very small and fluctuating. In the present tests, we used air flow instead of water flow for convenience.
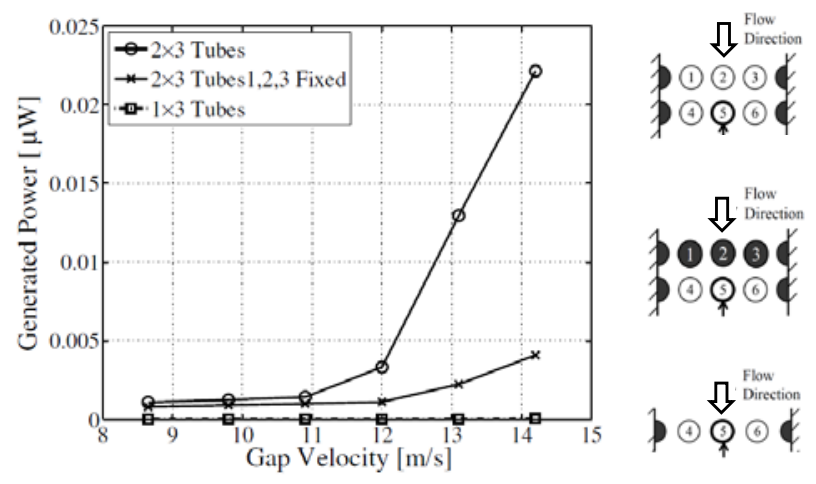

Fig. 12 Relationship between generated electric power and flow velocity for the cases 1, 2 and 6 (•: clamped tube)

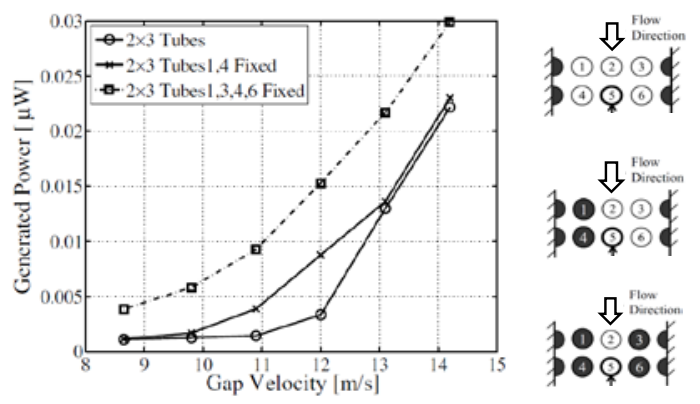

Fig. 13 Relationship between generated electric power and flow velocity for the cases 1, 4 and 5 (•: clamped tube)

Fluid density of the air is $1 / 1000$ th of that of water, thus the excitation force is very small that consequently leads to the very small generated energy. Moreover, the tubes 
used in the tests are very small such as the diameter of $20[\mathrm{~mm}]$. In the actual systems, very large tubes, such as 1 [m] in diameter and 5 [m] in length, can be employed. Employing this system, the estimation based on the tests results and the assumption on the generating efficiency of $30 \%$ gives the generated electric power up to around 600 $[\mathrm{W} / \mathrm{h}]$ as for a single tube. In addition, the present test results indicates that only two tubes located tandemly among $2 \times 3$ array with the other tubes clamped will give the largest generated electric power.

\section{Conclusions}

From the test tests utilizing air flow and very small tubes, it was found that the proposed energy harvesting system could generate electric power. However, the generated electric power is very small because of the small test tube model and small fluid density of air flow. Thus the results obtained here seem to have a less accuracy and reliability. Therefore, as the future tasks, tests utilizing water flow and large scale tube arrays are needed in developing the actual systems. And in addition, total generated electric power should be examined when multiple tube array units are employed.

\section{References}

[1] The executive committee of ocean energy systems, 2014 Annual report of OES (2014).

[2] Kanki, H. et al., "Research on Improvement in Efficiency of Wave Power Generation System by Applying Gyroscopic Moment", Proc. of 85th Annual Conf. of JSME Kansai Branch, No. 104-1, p. 9-3 (2010) (In Japanese).

[3] Kyozuka, Y., et al., "Hydrodynamic Characteristics of a Darrieus Water Turbine for Tidal Power Generation", Journal of the Japan Society of Naval Architects and Ocean Engineers, Vol. 4, pp. 39-45 (2006) (In Japanese).

[4] Chen, S. S., Flow-Induce Vibration of Circular Cylindrical Structures, Hemisphere Publishing Corp. (1987).

[5] Fujita, K., Ito, T. and Kohno, N., "Experimental Study on the Vibration of Circular Cylinders Subjected to Cross-Flow Jetted from a Narrow Gap", Journal of Fluids and Structures, Vol. 4, pp. 99-124 (1990).

[6] Ito, T, et al., "Study on Energy Harvesting System Utilizing Fluid-elastic Vibration of a Tube Array Subjected to Fluid Flow", Proceedings of $8^{\text {th }}$ International Conference on Sustainability in Energy and Buildings, SEB-16, in Italy (2016) (to be appeared).

[7] Connors, H., J., "Vibration of Heat Exchanger Tube Arrays", ASME Journal of Mechanical Design, Vol. 100, pp. 347-353 (1978). 